\title{
Fulminant type 1 diabetes mellitus in Japanese children and adolescents: multi-institutional joint research of the Japanese Study Group of Insulin Therapy for Childhood and Adolescent Diabetes
}

\author{
Kentaro Shiga ${ }^{1)}$, Tatsuhiko Urakami ${ }^{2}$, Junichi Suzuki ${ }^{2)}$, Yasuhiro Igarashi ${ }^{3)}$, Hanako Tajima ${ }^{4}$, \\ Shin Amemiya ${ }^{5)}$ and Shigetaka Sugihara); The Japanese Study Group of Insulin Therapy for \\ Childhood and Adolescent Diabetes (JSGIT) \\ 1) Children's Medical Center, Yokohama City University Medical Center, Kanagawa 232-0024, Japan \\ 2) Department of Pediatrics, Nihon University School of Medicine, Tokyo 101-8309, Japan \\ 3) Igarashi Children's Clinic, Miyagi 981-3203, Japan \\ 4) Department of Pediatrics, Nippon Medical School, Tokyo 113-8602, Japan \\ 5) Department of Pediatrics, Saitama Medical University, Saitama 350-0495, Japan \\ 6) Department of Pediatrics, Tokyo Women's Medical University Medical Center East, Tokyo 116-8567, Japan
}

\begin{abstract}
Fulminant type 1 diabetes mellitus (FT1DM) is a subtype of type 1 diabetes mellitus characterized by a remarkably abrupt onset. In Japan, FT1DM accounts for approximately 20\% of acute-onset adult type 1 diabetes mellitus cases; however, reports of pediatric-onset FT1DM are rare. We aimed to determine the frequency and clinical characteristics of FT1DM in Japanese children and adolescents by conducting a 2-phase questionnaire survey among the members of the Japanese Study Group of Insulin Therapy for Childhood and Adolescent Diabetes (JSGIT) regarding their clinical experience with FT1DM. Responses were obtained from 54 of the 79 participating hospitals (68.4\%). Of these, 8 hospitals managed a total of 15 pediatric patients with FT1DM (4 patients in each of 2 hospitals, 2 patients in 1 hospital, and 1 patient in each of 5 hospitals). The distribution of patient age was biphasic, with peaks in children younger than 5 years and older than 8 years of age. The clinical characteristics of FT1DM in this population (such as the duration from onset of symptoms to diagnosis, severity of symptoms, preceding flu-like episodes, and abnormal laboratory data) did not differ from those of patients with adult-onset FT1DM. The frequency of pediatric-onset FT1DM is low compared with that of adult-onset FT1DM. The genetic background and susceptibility patterns of pediatric patients with FT1DM may differ from those typical of adults with FT1DM, but both age groups share similar clinical characteristics.
\end{abstract}

Key words: Fulminant type 1 diabetes mellitus, Pediatric-onset diabetes mellitus, Child, Adolescent

IN JAPAN, patients with type 1 diabetes mellitus (T1DM) form a clinically heterogeneous group; the condition is divided into the following 3 subtypes: acuteonset, fulminant, and slowly progressive [1-3]. Imagawa et al. were the first to report on fulminant type 1 diabetes mellitus (FT1DM) in Japan [4]. The clinical characteris-

Submitted Feb. 1, 2018; Accepted Apr. 16, 2018 as EJ18-0029

Released online in J-STAGE as advance publication May 22, 2018

Correspondence to: Tatsuhiko Urakami, M.D., Ph.D., Department of Pediatrics, Nihon University Hospital, 1-6 Kandasurugadai, Chiyoda-ku, Tokyo 101-8309, Japan.

E-mail: urakami.tatsuhiko@nihon-u.ac.jp tics of FT1DM are defined as follows: Occurrence of diabetic ketosis or ketoacidosis soon (within approximately 7 days) after the onset of hyperglycemic symptoms; plasma glucose level $\geq 16.0 \mathrm{mmol} / \mathrm{L}$ ( $\geq 288 \mathrm{mg} / \mathrm{dL}$ ) and glycated hemoglobin (HbA1c) level $<8.5 \%$ (based on the Japan Diabetes Society [JDS] criteria) at first visit; and urinary C-peptide excretion $<10 \mu \mathrm{g} /$ day or fasting serum C-peptide level $<0.3 \mathrm{ng} / \mathrm{mL}(<0.10 \mathrm{nmol} / \mathrm{L})$ and $<0.5 \mathrm{ng} / \mathrm{mL}(<0.17 \mathrm{nmol} / \mathrm{L})$ after intravenous glucagon (or after meal) load at onset [5]. In addition, FT1DM has the following characteristics: Islet cell-related autoantibodies such as anti-glutamic acid decarboxylase 
antibodies (GADA) are absent in most cases; serum pancreatic enzyme levels (amylase, lipase, or elastase-1) are elevated in $98 \%$ of patients; and preceding flu-like symptoms (fever and upper respiratory symptoms, among others) or gastrointestinal symptoms (upper abdominal pain and nausea and/or vomiting, among others) are observed in $70 \%$ of patients [5].

The incidence of T1DM is not as high in Asian countries as it is in European countries and the in the US. In contrast, the incidence of FT1DM is higher in Asian than in European countries [6-10]. In Japan, FT1DM accounts for approximately $20 \%$ of acute-onset adult T1DM cases with ketosis at disease onset [6], but few reports have described cases of FT1DM in pediatric patients [10, 11], even though T1DM is more common among children and adolescents than among adults. This study aimed to clarify the frequency of FT1DM and its characteristics in Japanese children and adolescents.

\section{Materials and Methods}

\section{Respondents and patient cohort}

A 2-phase questionnaire survey was sent to the members of the Japanese Study Group of Insulin Therapy for Childhood and Adolescent Diabetes (JSGIT), established in 1994 [12]. This group now comprises pediatric diabetologists from 79 institutions (current chairman: Sugihara S.) and is the largest group studying pediatric T1DM in Japan. The $4^{\text {th }}$ research cohort of JSGIT has been underway since 2013, and 1,076 patients are registered in this cohort.

\section{Questionnaires}

In this 2-phase survey, the first questionnaire aimed to confirm whether the pediatric diabetologist had treated a case of FT1DM with an age of diagnosis below 16 years. The diagnosis of FT1DM was based on the diagnostic criteria described by the JDS in 2012 [5].

The second questionnaire was sent to those members who had clinical experience of managing FT1DM in pediatric patients. It included questions regarding the following aspects: patient's sex, age at FT1DM onset, body weight and height, duration from the onset of symptoms to diagnosis, symptoms at the time of onset, family history of diabetes, precursor symptoms (i.e., upper respiratory symptoms and/or gastrointestinal symptoms), blood glucose and $\mathrm{HbAlc}$ values at onset, existence of ketosis and/or ketoacidosis, blood and/or urine C-peptide immunoreactivity (CPR) values, presence of islet cell-related autoantibodies, concentrations of exocrine pancreatic enzymes, and human leukocyte antigen (HLA) class II typing.

\section{Data collection and measurements}

The clinical examination of each patient was conducted by the attending clinician at each institution. All values are presented as the mean \pm SD. JDS-based HbA1c values were converted to the $\mathrm{HbAlc}$ values recommended by the National Glycohemoglobin Standardization Program (NGSP) and the International Federation of Clinical Chemistry (IFCC) using the following equations: NGSP $(\%)=1.02 \times \operatorname{JDS}(\%)+0.25[13]$, and IFCC $(\mathrm{mmol} / \mathrm{mol})=(10.93 \times \mathrm{NGSP})-23.50$ [14], respectively.

Questionnaire response forms were collected by facsimile or e-mail, and were kept by the JSGIT secretariat. All data were analyzed by the authors.

\section{Ethical approval}

This study was approved by the review board of each participating institution in accordance with the ethical guidelines and regulations of the Declaration of Helsinki. Written consent was obtained from all patients or their legal guardians.

\section{Results}

\section{Data collection}

Responses to the questionnaires were obtained from 54 of 79 hospitals $(68.4 \%)$. Overall, 8 hospitals had treated a total of 15 pediatric patients with FT1DM (4 patients in each of 2 hospitals, 2 patients in 1 hospital, and 1 patient in each of 5 hospitals).

\section{Clinical characteristics of the patients}

The clinical characteristics of the patients are shown in Table 1 . The 15 patients included 6 boys and 9 girls, aged $9.2 \pm 5.1$ (range: 0.9-15.7) years. Six (40.0\%) patients ( 1 with T1DM and 5 with type 2 diabetes mellitus) each had a family history of diabetes. The mean interval from the onset of symptoms to diagnosis was 3.2 \pm 1.5 (range: $2-7$ ) days. At the onset of the disease, 9 $(60.0 \%)$ patients showed disturbances of consciousness, and $13(86.7 \%)$ experienced at least one symptom of upper respiratory tract infection or gastroenteritis preceding the onset of hyperglycemic symptoms.

The age at onset appeared to have a biphasic distribution, with 1 group including 5 younger children aged $<5$ 
Table 1 Clinical characteristics of the patients with fulminant type 1 diabetes mellitus

\begin{tabular}{|c|c|c|c|c|c|c|c|c|}
\hline $\begin{array}{l}\text { Case } \\
\text { No. }\end{array}$ & Sex & $\begin{array}{c}\text { Age } \\
\text { (years) }\end{array}$ & $\begin{array}{l}\text { BMI-SD } \\
\text { score }\end{array}$ & Symptoms & DKA/ketosis & $\begin{array}{c}\text { Duration from } \\
\text { onset (days) }\end{array}$ & Family history & Flu-like episode \\
\hline 1 & $\mathrm{~F}$ & 0.9 & -2.42 & Not doing well & $(+)$ & 3 & $(-)$ & $(-)$ \\
\hline 2 & $\mathrm{~F}$ & 1.8 & -0.93 & DC & $(+)$ & 5 & $(-)$ & $(+)$ \\
\hline 3 & M & 3 & -1.36 & $\mathrm{PD}, \mathrm{PU}$ & $(+)$ & 3 & (+) T1DM & $(+)$ \\
\hline 4 & $\mathrm{~F}$ & 3.5 & -2.34 & PD, PU, not doing well & $(+)$ & 7 & $(-)$ & $(+)$ \\
\hline 5 & $\mathrm{~F}$ & 5 & 0.42 & $\mathrm{VO}, \mathrm{DC}$ & $(+)$ & 2 & $(+) \mathrm{T} 2 \mathrm{DM}$ & $(+)$ \\
\hline 6 & M & 8 & -0.32 & PD, PU, VO, AP & $(+)$ & 2 & $(+) \mathrm{T} 2 \mathrm{DM}$ & $(+)$ \\
\hline 7 & M & 9.4 & -0.79 & PD, PU, fatigue & $(+)$ & (not recorded) & $(+) \mathrm{T} 2 \mathrm{DM}$ & $(-)$ \\
\hline 8 & M & 11.5 & -0.29 & $\mathrm{PD}, \mathrm{PU}, \mathrm{VO}, \mathrm{AP}, \mathrm{DC}$ & $(+)$ & 3 & $(+) \mathrm{T} 2 \mathrm{DM}$ & $(+)$ \\
\hline 9 & $\mathrm{~F}$ & 12 & -2.51 & PD, PU, DC & $(+)$ & 2 & $(-)$ & $(+)$ \\
\hline 10 & M & 12.1 & -0.18 & $\mathrm{PD}, \mathrm{PU}, \mathrm{VO}, \mathrm{AP}$ & $(+)$ & 4 & $(-)$ & $(+)$ \\
\hline 11 & $\mathrm{~F}$ & 12.5 & 0.34 & PD, PU, DC & $(+)$ & 5 & (+) $\mathrm{T} 2 \mathrm{DM}$ & $(+)$ \\
\hline 12 & $\mathrm{~F}$ & 13.4 & -0.73 & $\mathrm{VO}, \mathrm{DC}$ & $(+)$ & 3 & $(-)$ & $(+)$ \\
\hline 13 & F & 14.8 & -0.31 & $\mathrm{PD}, \mathrm{PU}, \mathrm{VO}, \mathrm{AP}, \mathrm{DC}$ & $(+)$ & 2 & $(-)$ & $(+)$ \\
\hline 14 & M & 15 & -1.13 & (not recorded) & $(+)$ & 2 & $(-)$ & $(+)$ \\
\hline 15 & $\mathrm{~F}$ & 15.7 & -1.21 & $\mathrm{VO}, \mathrm{RD}, \mathrm{DC}$ & $(+)$ & 2 & $(-)$ & $(+)$ \\
\hline
\end{tabular}

AP, abdominal pain; DC, disturbance of consciousness; PD, polydipsia; PU, polyuria; RD, respiratory disturbance; T1DM, type 1 diabetes mellitus; T2DM, type 2 diabetes mellitus; VO, vomit.

years and the other comprising 10 older children and adolescents aged $>8$ years (Fig. 1).

\section{Laboratory data at onset}

The laboratory data of all 15 patients are shown in Table 2. The mean blood glucose level at onset was $797.0 \pm 297.0$ (range: $409-1,134) \mathrm{mg} / \mathrm{dL}$ and the mean HbAlc value was $6.7 \pm 0.8$ (range: 5.6-8.3) \% [49.8 \pm 9.3 (range: $37.7-67.2$ ) $\mathrm{mmol} / \mathrm{mol}$ ]. The mean serum and urine CPR levels were $0.20 \pm 0.13$ (range: $0.02-$ $0.39) \mathrm{ng} / \mathrm{mL}$ and $4.4 \pm 3.4$ (range: $0.3-9.0) \mu \mathrm{g} /$ day, respectively. The mean serum levels of exocrine pancreatic enzymes were elevated in all 8 patients who had such measurements performed: Amylase, $413.4 \pm 326.2 \mathrm{IU} / \mathrm{L}$ (range: 94-879 IU/L); lipase, $119.3 \pm 98.0 \mathrm{IU} / \mathrm{L}$ (range: 16-219 IU/L); and elastase-1, $792.7 \pm 888.6 \mathrm{ng} / \mathrm{dL}$ (range: $168-1,810 \mathrm{ng} / \mathrm{dL}$ ). The results of radioimmunoassay testing for GADA were positive $(\geq 1.5 \mathrm{U} / \mathrm{mL})$ in 4 (26.7\%) cases, but the mean titer was relatively low at $5.5 \pm 3.6$ (range: $3.0-9.7$ ) U/mL.

HLA type was analyzed in 12 patients. The HLA class II haplotype was identified in 8 of these 12 patients; 4 had DRB1*04:05-DQB1*04:01 and 3 had DRB1*09:01DQB1*03:03. Overall, 6 of 8 patients had HLA DRB1*
04:05-DQB1*04:01 and/or DRB1*09:01-DQB1*03:03 (Table 3).

\section{Discussion}

In Japan, a nation-wide survey showed that FT1DM was diagnosed in $19.4 \%$ of patients with acute-onset adult T1DM with ketosis at disease onset. The mean age at onset was 39.1 years while the proportion aged younger than 20 years at onset was less than $10 \%$; however, the frequency of FT1DM among pediatric patients was unclear [6]. Recent studies suggest that FT1DM is common in eastern Asian countries such as Japan, Korea, and China. In these countries, most FT1DM patients are adults [8-10]; few reports have focused on FT1DM in the pediatric population $[10,11]$.

In the present study, 16 patients aged $<16$ years fulfilled the diagnostic criteria for FT1DM. The $4^{\text {th }}$ research cohort of the JSGIT has been under way since 2013. Currently, 1,076 patients, including 450 boys and 629 girls aged $6.5 \pm 3.8$ years (range: $0.1-18.3$ years), are registered in this cohort. A sizeable proportion of pediatric FT1DM cases in Japan were, thus, included in the present study. The time of onset was not restricted in this 


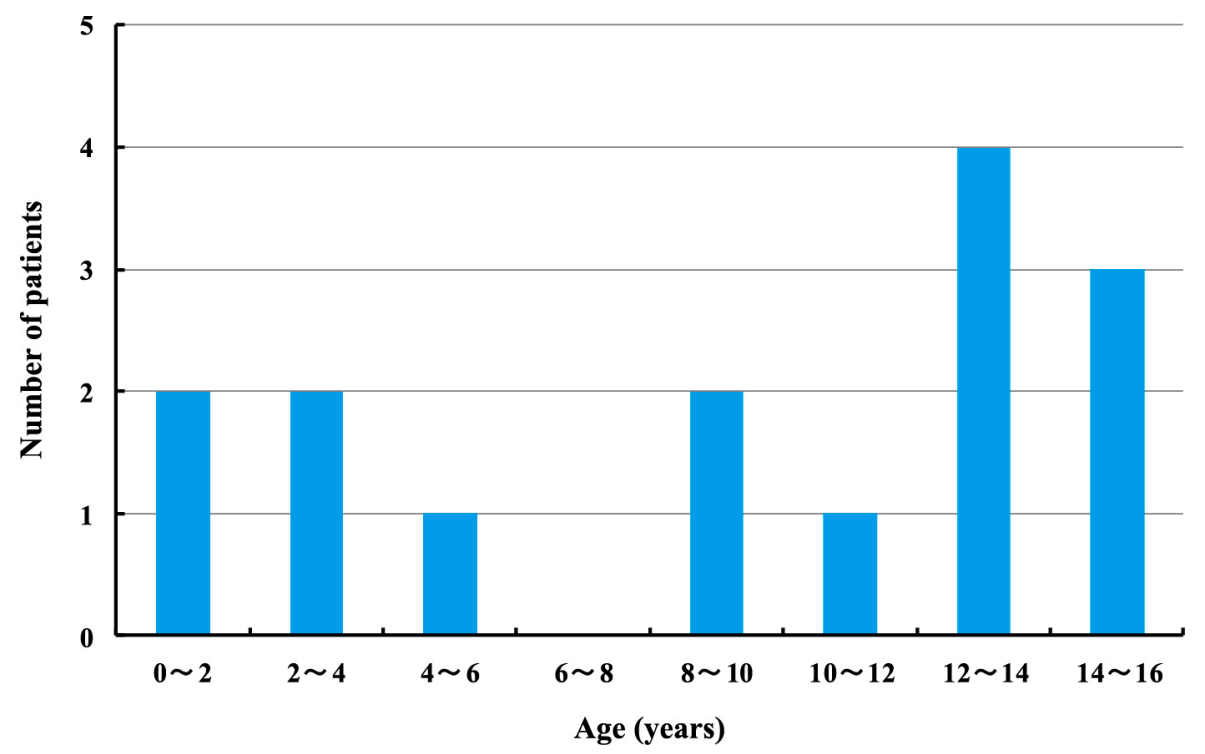

Fig. 1 Age distribution of children with fulminant type 1 diabetes mellitus

The age at onset was distributed biphasically: One group comprised younger children $<5$ years of age, the other comprised children and adolescents $>8$ years of age.

Table 2 The laboratory data of all patients with fulminant type 1 diabetes mellitus

\begin{tabular}{|c|c|c|c|c|c|c|c|}
\hline Case No. & $\begin{array}{l}\text { Glucose } \\
(\mathrm{mg} / \mathrm{dL})\end{array}$ & $\begin{array}{l}\mathrm{HbA} 1 \mathrm{c} \\
(\%)\end{array}$ & $\begin{array}{c}\mathrm{HbA} 1 \mathrm{c} \\
(\mathrm{mmol} / \mathrm{mol})\end{array}$ & $\begin{array}{l}\text { S-CPR } \\
(\mathrm{ng} / \mathrm{mL})\end{array}$ & $\begin{array}{l}\text { U-CPR } \\
(\mu \mathrm{g} / \text { day })\end{array}$ & GADA & $\begin{array}{c}\text { Elevation of } \\
\text { pancreatic enzymes }\end{array}$ \\
\hline 1 & 679 & 8.3 & 67.2 & 0.36 & 3.8 & $(+) ; 3.0$ & $(+)$ \\
\hline 2 & 565 & 7.9 & 64.8 & $<0.3$ & n.e. & $(-)$ & n.e. \\
\hline 3 & 409 & 7 & 53 & 0.2 & 3.9 & $(+)$ & n.e. \\
\hline 4 & 772 & 8 & 63.9 & $<0.3$ & 7 & $(-)$ & n.e. \\
\hline 5 & 618 & 5.9 & 41 & 0.17 & n.e. & $(-)$ & $(+)$ \\
\hline 6 & 749 & 6.3 & 45.4 & 0.1 & 4.6 & $(-)$ & $(+)$ \\
\hline 7 & 626 & 5.9 & 41 & $<0.5$ & 0.6 & $(+) ; 9.7$ & n.e. \\
\hline 8 & 843 & 6.8 & 50.8 & 0.3 & n.e. & $(-)$ & $(+)$ \\
\hline 9 & 949 & 6.7 & 49.7 & $<0.2$ & 6.1 & $(+) ; 3.8$ & n.e. \\
\hline 10 & $>500$ & 5.6 & 37.7 & $<0.02$ & 0.1 & $(-)$ & n.e. \\
\hline 11 & 708 & 7 & 53 & $<0.3$ & 9 & $(-)$ & n.e. \\
\hline 12 & 1,540 & 6 & 42.1 & 0.09 & 0.3 & $(-)$ & $(+)$ \\
\hline 13 & 1,070 & 6.6 & 48.6 & 0.02 & n.e. & $(-)$ & $(+)$ \\
\hline 14 & 496 & 6.5 & 47.5 & 0.39 & 9 & $(-)$ & $(+)$ \\
\hline 15 & 1,134 & 5.9 & 41 & 0.06 & n.e. & $(-)$ & $(+)$ \\
\hline
\end{tabular}

GADA, glutamic acid decarboxylase antibodies; HbAlc, glycated hemoglobin; n.e. not examined; S-CPR, serum C-peptide immunoreactivity; U-CPR, urine C-peptide immunoreactivity. 
Table 3 Results of HLA typing ${ }^{a}$

\begin{tabular}{cccc}
\hline Case No. & Age (years) & HLA typing & GADA \\
\hline 1 & 0.9 & DRB1*04:05-DQB1*04:01/DRB1*09:01-DQB1*03:03 & $(+)$ \\
\hline 3 & 3 & DRB1*01:01-DQB1*05:01/DRB1*09:01-DQB1*03:03 & $(+)$ \\
\hline 6 & 8 & DRB1*04:05-DQB1*04:01/DRB1*16:02-DQB1*05:02 & \\
\hline 7 & 9.4 & DRB1*03:01-DQB1*02:01/DRB1*16:02-DQB1*05:02 & $(+)$ \\
\hline 8 & 11.5 & DRB1*15:02-DQB1*06:01/DRB1*09:01-DQB1*03:03 \\
\hline 12 & 13.4 & DRB1*04:05-DQB1*04:01/DRB1*08:02-DQB1*04:02 \\
\hline 13 & 14.8 & DRB1*11:01-DQB1*03:01/DRB1*14:05-DQB1*05:03 \\
\hline 15 & 15.7 & DRB1*04:05-DQB1*04:01/DRB1*04:05-DQB1*04:01 \\
\hline
\end{tabular}

a The HLA class II haplotype was identified in 8 patients.

GADA, glutamic acid decarboxylase antibodies; HLA, human leukocyte antigen.

study; therefore, patients not registered in the $4^{\text {th }}$ research cohort were also included. Because it was difficult to determine what denominator to use, we could not calculate the exact prevalence and incidence of pediatric-onset FT1DM. Nevertheless, the frequency of FT1DM is estimated to be considerably lower in children and adolescents than in adults. One reason why pediatric-onset FT1DM is considered rare may be that it is unfamiliar to pediatricians; hence, some cases of FT1DM may not be identified.

With regard to patient age at disease onset, the distribution seems to be biphasic, with 1 group comprising younger children ( $<5$ years old) and the other comprising older children and adolescents $(>8$ years old). Although all patients met the diagnostic criteria for FT1DM, younger patients had relatively higher HbAlc levels and lower body mass index-standard deviation scores than older patients.

The currently used diagnostic criteria for FT1DM are mainly based on adult data [5], wherein proof of an abrupt onset depends on the lack of a substantial elevation in the value of HbAlc. However, in infant patients, the percentage of fetal hemoglobin $(\mathrm{HbF})$ in the blood is estimated to be high, and a higher percentage of non$\mathrm{HbAlc}$, such as $\mathrm{HbF}$, is associated with a lower level of HbAlc [15]. Therefore, in the present study, the true duration of high blood glucose status may have been underestimated by the relatively low $\mathrm{HbAlc}$ values. Moreover, it may be more difficult to establish the diagnosis of FT1DM in younger patients.

The mean serum and urine CPR levels observed in the present study were both at an extremely low level, indicating very low secretion of endogenous insulin. The exact pathophysiology resulting in the onset of FT1DM is unknown. The sudden and complete destruction of pancreatic $\beta$-cells is thought to be a cause of this disease, but the precise mechanisms of such cell destruction remain unclear. It has been suggested that viral infections, such as reactivation of human herpes virus-6, Epstein-Barr virus, cytomegalovirus, coxsackievirus, mumps virus, and influenza type B virus, are related to the onset of FT1DM [16-22]. In this study, $86.7 \%$ of patients presented with signs suggestive of a preceding viral infection. Urakami et al. reported that patients with type 1B diabetes mellitus were significantly younger at the time of diagnosis and had a higher frequency of a preceding viral infection than those with type 1A diabetes mellitus [23]. One possible mechanism underlying FT1DM is that in some susceptible individuals, viral infection initiates an abnormal immune response that triggers abrupt and total $\beta$-cell destruction [24-26]. Immunologic immaturity against viral infection may also be associated with the onset of FT1DM in these populations. Because of the absence of insulitis and islet-related autoantibodies, FT1DM was first reported as a subtype of idiopathic (type 1B) diabetes mellitus [4]. However, recent studies show that T-cells, macrophages, and dendritic cells infiltrate into the islets or exocrine tissues around the islets soon after the onset of the disease. Enterovirus capsid protein 1 and enterovirus RNA has been detected in islet cells and exocrine tissues [27, 28]. Enterovirus infection of islet cells might induce innate immunity $[29,30]$. Interferon- $\alpha$ and $\beta$, expressed in islet cells and infiltrating mononuclear cells, produce autoimmunity to islet cells including $\beta$ - and $\alpha$-cells; this leads to rapid and complete destruction of islet cells in patients 
with FT1DM [27, 31].

Individual susceptibility may be defined by various potential factors, including genetic factors, especially HLA class II [32-35]. With regard to HLA class II typing, among the 8 patients in whom the HLA class II haplotype was evaluated, DRB1*04:05-DQB $1 * 04: 01$ was identified in 4 cases and DRB1*09:01-DQB $1 * 03: 03$ was identified in 3 cases. Both DRB1*04:05-DQB1* 04:01 and DRB1*09:01-DQB1*03:03 are related to both acute onset and slowly progressive T1DM. In particular, DRB1*09:01-DQB1*03:03 is more strongly associated with GADA-positive T1DM, while DRB1*04:05-DQB1* 04:01 is more strongly associated with FT1DM. High frequency of homozygous haplotype of DRB1*04:05DQB1*04:01 is reported in adult-onset FT1DM, which may induce greater genetic effect on the destruction of $\beta$-cells, and is supposed to play an important role in the development of FT1DM [31-33]. In this study, only one patient had homozygous haplotype of DRB1*04:05DQB1*04:01. The number of patients who underwent HLA typing was too small for appropriate evaluation, so it is unclear whether the frequency of homozygous haplotype of DRB1*04:05-DQB1*04:01 is actually low in young-onset FT1DM.

On the other hand, a high frequency of DRB $1 * 09: 01$ DQB1*03:03 haplotype, which is typically associated with autoimmune T1DM, has also been demonstrated in pregnancy-associated FT1DM [36]. Moreover, recent studies have indicated an association between DRB1* 09:01-DQB1*03:03 and FT1DM [35].

Islet-cell related autoantibodies, such as GADA, are generally undetectable in patients with FT1DM. However, a nation-wide survey showed that approximately $5 \%$ of patients with FT1DM test positive for GADA, although in most of such cases, the titer is low and transient $[6,37]$. In the present study, 4 cases $(26.7 \%)$ yielded positive results for GADA. This percentage seems relatively high compared with findings from previous reports of adult patients [6]. However, the mean GADA titer was relatively low $(5.5 \pm 3.6 \mathrm{U} / \mathrm{mL})$, similar to that in adult patients. In this study 2 of 4 GADA-positive patients had the DRB1*09:01-DQB1*03:03 HLA class II haplotype; both were $<3$ years of age. It seems that autoimmune mechanisms, rather than genetic mechanisms, may play an important role in such patients. In pediatric patients, particularly in younger children, although the clinical course of FT1DM is similar to that seen in adults, the genetic background and mechanisms underlying susceptibility to FT1DM may be different.
The present study has some limitations. It was a retrospective, multi-institutional joint research project and clinical examinations were conducted individually in each institution; therefore, all data at onset were not necessarily provided. Because the number of FT1DM patients in this study was so small, we could not perform a statistically meaningful analysis; the accumulation of cases and further investigations are warranted.

In conclusion, this study demonstrates that the estimated frequency of FT1DM is considerably lower in pediatric than in adult patients. Although the genetic background and susceptibility patterns of childhoodonset FT1DM may differ from those typical of adultonset FT1DM, the clinical characteristics of FT1DM are similar in both pediatric and adult patients. Pediatricians should recognize the existence of FT1DM as it is a lifethreatening metabolic state for which an accurate diagnosis, made as early as possible, and provision of appropriate therapy, are crucial, especially in pediatric patients.

\section{Acknowledgements}

This study was supported by the Japan Diabetes Foundation.

The Japanese Study Group of Insulin Therapy for Children and Adolescent Diabetes (JSGIT) comprised the following members: Ikuko Takahashi, Akita University School of Medicine; Yusuke Tanahashi, Kumihiro Matsuo, Asahikawa Medical University; Jiro Iwamoto, Shuichi Yatsuga, Aso-Iizuka Hospital; Yasuhiro Igarashi, Igarashi Children's Clinic; Akira Endo, Iwata City Hospital; Koji Takemoto, Junpei Hamada, Ehime University School of Medicine; Kenji Ihara, Oita University Faculty of Medicine Graduate School of Medicine; Yukashi Ohki, Ohki Pediatric Endocrinology and Metabolism Clinic; Ryuzo Takaya, Osaka Medical College; Takahiro Mochizuki, Osaka Medical College; Tomoyuki Kawamura, Yuko Horita, Osaka City University School of Medicine; Mahoko Furujo, Okayama Medical Center; Masaru Inoue, Okayama Red Cross General Hospital; Kosei Hasegawa, Okayama University School of Medicine; Masanori Adachi, Kanagawa Children's Medical Center; Michiko Okajima, Kanazawa University School of Medicine; Kazuhiro Shimura, Kawasaki Municipal Hospital; Shigeyuki Ohtsu, Kazuteru Kitsuda, Kitasato University School of Medicine; Yoshiya Ito, Kitami Red Cross Hospital; Tomohiro Hori, Gifu University Hospital; Kanako Ishii, Kyushu University School of Medicine; Yasusada Kawada, Kyushu Rosai Hospital; 
Hisakazu Nakajima, Ikuyo Ito, Kyoto Prefectural University of Medicine; Kimitoshi Nakamura, Kumamoto University School of Medicine; Shinichiro Miyagawa, National Hospital Organization Kure Medical Center; Kazuhiko Jinno, Hiroshima Prefectural Hospital; Reiko Horikawa, Tomoko Yoshida, Maki Fukami, National Center for Child Health and Development; Testuo Mori, Shinshu Ueda Medical Center; Kisyo Kobayashi, Kobayashi Kids Clinic; Susumu Konda, Konda Children's Clinic; Nozomu Sasaki, Kawagoe Clinic of Saitama Medical University; Shin Amemiya, Toru Kikuchi, Ikuma Musha, Saitama Medical University; Katsuya Aizu, Hiroshi Mochizuki, Saitama Children's Medical Center; Tomoyuki Hotsubo, Sapporo Pediatric Endocrinology Clinic; Kohei Sato, Sapporo Factory Kids Clinic; Yukiyo Yamamoto, University of Occupational and Environmental Health; Hiroko Kadowaki, Sanno Hospital; Aki Nishii, JR Sendai Hospital; Katsuyuki Matsui, Shiga Medical School; Ichiro Yokota, Tatsuya Miyoshi, Shikoku Medical Center for Children and Adults; Noriyuki Takubo, Juntendo University of Medicine; Yosuke Hara, Shinshu University Hospital; Nobuo Matsuura, Seitoku University; Shigeki Miyamoto, Seitoku University Junior College; Shun Soneda, St. Marianna University School of Medicine; Toshikazu Takahashi, Takahashi Clinic; Kohji Tsubouchi, Chuno Kosei Hospital; Toshi Tatematsu, Chubu Rosai Hospital; Kanshi Minamitani, Teikyo University Chiba Medical Center; Eishin Ogawa, Yoichi Izumi, Teikyo University School of Medicine; Goro Sasaki, Tokyo Dental College Ichikawa General Hospital; Emiko Tachikawa, Tokyo Women's Medical University; Yasuko Uchigata, Tokyo Women's Medical University School of Medicine; Shigetaka Sugihara, Yuki Yasuda, Tokyo Women's Medical University Medical Center East; Kaori Sasaki,
Tokyo Women's Medical University Yachiyo Medical Center; Sachiko Kitanaka, Tsuyoshi Isojima, Tokyo University School of Medicine; Ikuma Fujiwara, Junko Kanno, Tohoku University School of Medicine; Yumiko Kotani, Tokushima University School of Medicine; Tadayuki Ayabe, Dokkyo Medical University Koshigaya Hospital; Naoto Shimura, Junko Ichikawa, Dokkyo Medical University; Keiichi Hanaki, Tottori Prefectural Kousei Hospital; Junichi Nagaishi, Tottori Municipal Hospital; Susumu Kanzaki, Masanobu Fujimoto, Tottori University Faculty of Medicine; Junko Ito, Toranomon Hospital; Haruo Mizuno, Nagoya City University Hospital; Yuuki Abe, Niigata City General Hospital; Keisuke Nagasaki, Yohei Ogawa, Niigata University School of Medicine; Shinji Kadotani, Yoneo Kashihara, Nishinomiya Municipal Central Hospital; Hanako Tajima, Nippon Medical School; Tatsuhiko Urakami, Junichi Suzuki, Misako Okuno, Nihon University Hospital; Tsutomu Ogata, Konosuke Ohtaka, Hamamatsu University School of Medicine; Kenichi Miyako, Fukuoka Children's Hospital; Hitoshi Kohno, Fukuoka Tokushukai Hospital; Takao Fujisawa, Shigeru Suga, National Mie Hospital; Akemi Koike, Koike Child Clinic; Shoji Nakayama, Mominoki Hospital; Koji Kobayashi, Hideaki Yagasaki, Yamanashi University School of Medicine; Mie Mochiduki, Yamanashi University School of Medicine; Rika Kizu, Yokosuka Kyosai Hospital; Kentaro Shiga, Yokohama City University Medical Center; and Nobuyuki Kikuchi, Yokohama Minato Red Cross Hospital.

\section{Disclosure}

The authors declare no conflicts of interest concerning this manuscript.

\section{References}

1. Kawasaki E, Eguchi K (2004) Is type 1 diabetes in the Japanese population the same as among Caucasians? Ann NY Acad Sci 1037: 96-103.

2. Seino Y, Nanjo K, Tajima N, Kadowaki T, Kashiwagi A, et al. (2010) Report of the committee on the classification and diagnostic criteria of diabetes mellitus. $J$ Diabetes Investig 1: 212-228.

3. Kawasaki E, Maruyama T, Imagawa A, Awata T, Ikegami $\mathrm{H}$, et al. (2014) Diagnostic criteria for acute-onset type 1 diabetes mellitus (2012): report of the committee of Japan Diabetes Society on the research of fulminant and acute- onset type 1 diabetes mellitus. J Diabetes Investig 5: 115118.

4. Imagawa A, Hanafusa T, Miyagawa J, Matsuzawa Y, Osaka IDDM Study Group (2000) A novel subtype of type 1 diabetes mellitus characterized by a rapid onset and an absence of diabetes-related antibodies. $N$ Engl $\mathrm{J} \mathrm{Med}$ 342: 301-307.

5. Imagawa A, Hanafusa $T$, Awata $T$, Ikegami $H$, Uchigata $\mathrm{Y}$, et al. (2012) Report of the committee of the Japan diabetes society on the research of fulminant and acute-onset type 1 diabetes mellitus: new diagnostic criteria of fulmi- 
nant type 1 diabetes mellitus (2012). J Diabetes Investig 3: 536-539.

6. Imagawa A, Hanafusa T, Uchigata Y, Kanatsuka A, Kawasaki E, et al. (2003) Fulminant type 1 diabetes: a nationwide survey in Japan. Diabetes Care 26: 23452352.

7. Kida K, Mimura G, Ito T, Murakami K, Ashkenazi I, et al. (2000) Incidence of type 1 diabetes mellitus in children aged 0-14 in Japan, 1986-1990, including an analysis for seasonality of onset and month of birth: JDS study. Diabet Med 17: 59-63.

8. Cho YM, Kim JT, Ko KS, Koo BK, Yang SW, et al. (2007) Fulminant type 1 diabetes in Korea: high prevalence among patients with adult-onset type 1 diabetes. Diabetologia 50: 2276-2279.

9. Liu L, Mao J, Lu Z, Yan X, Ye Y, et al. (2012) Clinical analysis of fulminant type 1 diabetes in China and comparison with a nationwide survey in Japan. Diabetes Metab Syndr 6: 203-206.

10. Luo S, Zhang Z, Li X, Yang L, Lin J, et al. (2013) Fulminant type 1 diabetes: a collaborative clinical cases investigation in China. Acta Diabetol 50: 53-59.

11. Kim MS, Kim CJ, Ko CW, Hwang PH, Lee DY (2011) Fulminant type 1 diabetes mellitus in Korean adolescents. J Pediatr Endocr Met 24: 679-681.

12. Matsuura N, Yokota Y, Kazahari K, Sasaki N, Amemiya S, et al. (2001) The Japanese Study Group of Insulin Therapy for Childhood and Adolescent Diabetes (JSGIT): initial aims and impact of the family history of type 1 diabetes mellitus in Japanese children. Pediatr Diabetes 2: 160-169.

13. Kashiwagi A, Kasuga M, Araki E, Oka Y, Hanafusa T, et al. (2012) International clinical harmonization of glycated hemoglobin in Japan: from Japan Diabetes Society to National Glycohemoglobin Standardization Program values. J Diabetes Investig 3: 39-40.

14. Hoelzel W, Weykamp C, Jeppsson JO, Miedema K, Barr JR, et al. (2004) IFCC reference system for measurement of hemoglobin A1c in human blood and the national standardization schemes in the United States, Japan, and Sweden: a method-comparison study. Clin Chem 50: 166174.

15. Felner EI, McGrath M (2008) Inaccurate hemoglobin A1c levels in patients with type 1 diabetes and hereditary persistence of hemoglobin F. J Pediatr 153: 137-139.

16. Sekine N, Motokura T, Oki T, Umeda Y, Sasaki N, et al. (2001) Rapid loss of insulin secretion in a patient with fulminant type 1 diabetes mellitus and carbamazepine hypersensitivity syndrome. JAMA 285: 1153-1154.

17. Chiou CC, Chung WH, Hung SI, Yang LC, Hong HS (2006) Fulminant type 1 diabetes mellitus caused by drug hypersensitivity syndrome with human herpesvirus 6 infection. J Am Acad Dermatol 54: S14-S17.
18. Fujiya A, Ochiai H, Mizukoshi T, Kiyota A, Shibata T, et al. (2010) Fulminant type 1 diabetes mellitus associated with a reactivation of Epstein-Barr virus that developed in the course of chemotherapy of multiple myeloma. $J$ Diabetes Investig 1: 286-289.

19. Onuma H, Tohyama M, Imagawa A, Hanafusa $T$, Kobayashi T, et al. (2012) High frequency of HLA B62 in fulminant type 1 diabetes with the drug-induced hypersensitivity syndrome. J Clin Endocrinol Metab 97: E2277E2281.

20. Akatsuka H, Yano Y, Gabazza EC, Morser J, Sasaki R, et al. (2009) A case of fulminant type 1 diabetes with coxsackie B4 virus infection diagnosed by elevated serum levels of neutralizing antibody. Diabetes Res Clin Pract 84: e50-e52.

21. Goto A, Takahashi Y, Kishimoto M, Nakajima Y, Nakanishi K, et al. (2008) A case of fulminant type 1 diabetes associated with significant elevation of mumps titers. Endocr J 55: 561-564.

22. Sano H, Terasaki J, Tsutsumi C, Imagawa A, Hanafusa T (2007) A case of fulminant type 1 diabetes mellitus after influenza B infection. Diabetes Res Clin Pract 79: e8-e9.

23. Urakami $\mathrm{T}$, Inami I, Morimoto $\mathrm{S}$, Kubota $\mathrm{S}$, Owada $\mathrm{M}$ (2002) Clinical characteristics of non-immune-mediated, idiopathic type 1 (type 1B) diabetes mellitus in Japanese children and adolescents. J Pediatr Endocrinol Metab 15: 283-288.

24. Imagawa I, Hanafusa T (2006) Pathogenesis of fulminant type 1 diabetes. Rev Diabet Stud 3: 169-177.

25. Hanafusa T, Imagawa A (2008) Insulitis in human type 1 diabetes. Ann NY Acad Sci 1150: 297-299.

26. Imagawa A, Hanafusa $T$ (2011) Fulminant type 1 diabetes - an important subtype in East Asia. Diabetes Metab Res Rev 27: 959-964.

27. Tanaka S, Nishida Y, Aida K, Maruyama T, Shimada A, et al. (2009) Enterovirus infection, CXC chemokine ligand 10 (CXCL10), and CXCR3 circuit. Diabetes 58: 2285-2291.

28. Shibasaki S, Imagawa A, Tauriainen S, Iino M, Oikarinen M, et al. (2010) Expression of Toll-like receptors in the pancreas of recent-onset fulminant type 1 diabetes. Endocr J 57: 211-219.

29. Kobayashi T, Nishida Y, Tanaka S, Aida K (2011) Pathological changes in the pancreas of fulminant type 1 diabetes and slowly progressive insulin-dependent diabetes mellitus (SPIDDM): innate immunity in fulminant type 1 diabetes and SPIDDM. Diabetes Metab Res Rev 27: 965970.

30. Aida K, Nishida Y, Tanaka S, Maruyama T, Shimada A, et al. (2011) RIG-1- and MDA5-initiated innate immunity linked with adaptive immunity accelerates $\beta$-cell death in fulminant type 1 diabetes. Diabetes 60: 884-889.

31. Tanaka S, Aida K, Nishida Y, Kobayashi T (2013) Patho- 
physiological mechanisms involving aggressive islet cell destruction in fulminant type 1 diabetes. Endocr $J 60$ : 837-845.

32. Imagawa A, Hanafusa $T$, Uchigata $Y$, Kanatsuka A, Kawasaki E, et al. (2005) Different contribution of class II HLA in fulminant and typical autoimmune type 1 diabetes mellitus. Diabetologia 48: 294-300.

33. Tanaka S, Kobayashi T, Nakanishi K, Koyama R, Okubo M, et al. (2002) Association of HLA-DQ genotype in autoantibody-negative and rapid-onset type 1 diabetes. Diabetes Care 25: 2302-2307.

34. Kawabata $\mathrm{Y}$, Ikegami $\mathrm{H}$, Awata $\mathrm{T}$, Imagawa $\mathrm{A}$, Maruyama T, et al. (2009) Differential association of HLA with three subtypes of type 1 diabetes: fulminant, slowly progressive and acute-onset. Diabetologia 52:
2513-2521.

35. Tsutsumi C, Imagawa A, Ikegami H, Makino H, Kobayashi T, et al. (2012) Class II HLA genotype in fulminant type 1 diabetes: a nationwide survey with reference to glutamic acid decarboxylase antibodies. J Diabetes Investig 3: 62-69.

36. Shimizu I, Makino H, Imagawa A, Iwahashi H, Uchigata $\mathrm{Y}$, et al. (2006) Clinical and immunogenetic characteristics of fulminant type 1 diabetes associated with pregnancy. J Clin Endocrinol Metab 91: 471-476.

37. Hanafusa T, Imagawa A (2007) Fulminant type 1 diabetes: a novel clinical entity requiring special attention by all medical practitioners. Nat Clin Pract Endocrinol Metab 3: $36-45$. 